\title{
The Relation of Education and Income to Cognitive Function among Professional Women
}

\author{
Sunmin Lee ${ }^{\mathrm{a}}$ Julie E. Buring ${ }^{\mathrm{b}-\mathrm{d}}$ Nancy R. Cook $^{\mathrm{b}}$ Francine Grodstein ${ }^{\mathrm{c}-\mathrm{e}}$ \\ ${ }^{a}$ Department of Health and Behavioral Sciences, University of Colorado at Denver and Health Sciences Center, \\ Denver, Colo., 'b Department of Medicine, Division of Preventive Medicine, Brigham and Women's Hospital, \\ Harvard Medical School, ${ }^{c}$ Department of Epidemiology, Harvard School of Public Health, ${ }^{\mathrm{d}}$ Division of Aging \\ and ${ }^{\mathrm{e}}$ Channing Laboratory, Department of Medicine, Brigham and Women's Hospital, Harvard Medical School, \\ Boston, Mass., USA
}

\section{Key Words}

Educational attainment $\cdot$ Income $\cdot$ Cognitive function .

Cognitive decline

\begin{abstract}
We investigated the relation of educational attainment and annual household income to cognitive function and cognitive decline in community-dwelling women aged 66 years or older. Subjects were 6,314 health professionals participating in the Women's Health Study, among whom information on education and income was selfreported. From 1998 to 2000, we administered five cognitive tests, measuring general cognition, episodic memory and verbal fluency, using a validated telephone interview. Second cognitive assessments were conducted approximately 2 years later; information was complete for 5,573 women at the time of analysis, with $94 \%$ follow-up. We used linear and logistic regression to calculate multivariate-adjusted mean differences as well as odds of cognitive impairment (defined as worst $10 \%$ of test distribution) and of substantial decline in performance (worst $10 \%$ of distribution), across various levels of education and income. After adjusting for numerous potential confounding factors, we found strong trends of
\end{abstract}

increasing mean cognitive performance with increasing level of education ( $p$ trend $<0.0005$ on all cognitive measures). Odds of cognitive impairment also consistently decreased with increasing education. For income, we found significant trends of increasing mean cognitive performance with increasing income on the summary score and on episodic memory ( $p$ trends $<0.0001$ ). Results were generally similar for cognitive decline over 2 years, although slightly weaker. Thus, in these well-educated, professional women, educational attainment and income both predicted cognitive function and decline.

Copyright (C) 2006 S. Karger AG, Basel

\section{Introduction}

Numerous studies have indicated an association between educational attainment and both cognitive function and cognitive decline over time [1-9]. However, most of these studies have generally examined populations with somewhat low levels of education, and it is less clear whether this relation persists among more highly educated groups. In the only large-scale investigation of women with more than high school education, each higher level of education was still related to increasingly better

\section{KARGER}

(c) 2006 S. Karger AG, Basel

Fax +41613061234 E-Mail karger@karger.ch www.karger.com www.karger.com/ned
Sunmin Lee, ScD

Department of Health and Behavioral Sciences, University of Colorado at Denver and

Health Sciences Center, Campus Box 188, PO Box 173364

Denver, CO 80217-3364 (USA)

Tel. +1 303556 6793, Fax +1 303556 8501, E-Mail sunmin.lee@cudenver.edu 
cognitive function and less cognitive decline [8]. In this same study, however, there was no effect of census tract median household income or husband's educational attainment - suggesting that education may impact cognition independently of socioeconomic status; however, census tract as a marker of income is imperfect and thus this study may not have adequately considered income level.

To confirm the effects of education on cognitive function among highly educated subjects, and to further explore the potential impact of income, we investigated these issues among well-educated women enrolled in the Women's Health Study (WHS), a large cohort of female health professionals. In this cohort of health professionals, several important potential confounders, such as access to healthcare, are limited, thus enhancing the possibility for an especially valid estimation of relations between education, income and cognition.

\section{Methods}

\section{The WHS}

The WHS was a randomized, double-blind, placebo-controlled trial to test the benefits and risks of low-dose aspirin and vitamin E supplements for the prevention of cardiovascular disease and cancer [10]. From 1992 to 1996, investigators randomized 39,876 nurses who were free of cardiovascular disease and cancer to one of four treatment groups. Beginning in 1998, women aged 66 years or older participated in serial telephone assessments of cognitive function. Information on various health characteristics and behaviors were updated through annual mailed questionnaires, and follow-up of the cohort exceeded 93\%.

\section{Population for Analysis}

All WHS participants aged 66 years and older as of $1998(\mathrm{n}=$ 7,187 ), except 69 women who had a diagnosed stroke, were eligible to participate in the cognitive study. Of 7,118 women whom we attempted to contact and interview, 408 women $(5.7 \%)$ refused to participate and 396 women $(5.9 \%)$ were unreachable (i.e., no telephone number). Therefore 6,314 women completed the initial cognitive assessment. In general, women who did not participate in the cognitive study did not differ substantially from those who participated in terms of their level of education or income. For example, of those who participated, $33.4 \%$ had a bachelor's or advanced degree versus $35.1 \%$ among those who did not participate.

At the time of these analyses, follow-up interviews were almost complete; attempts to conduct second interviews (after a 2-year interval) had been made for 5,907 (93.5\%) of the 6,314 women who completed a baseline assessment. Of these women, $181(3.1 \%)$ refused, $132(2.3 \%)$ were lost to follow-up, and $21(0.4 \%)$ had died. Therefore we included 5,573 women in analyses of cognitive decline. Women with follow-up data on cognitive decline were generally similar to those without data on decline; for example, among the former, $34 \%$ had a bachelor's or advanced degree and among the latter, this figure was $31 \%$.

\section{Cognitive Function Assessment}

We assessed women's cognitive function using a validated telephone battery [8]. We administered the Telephone Interview of Cognitive Status (TICS) [11], which is modeled on the Mini-Mental State Examination (MMSE), the most frequently used test of cognitive function. Brandt et al. [11] reported a strong linear relation between the TICS and the MMSE (Pearson correlation =0.94). TICS scores can range from 0 to 41 , and the TICS includes 11 items, measuring orientation, immediate verbal memory (word list recall), opposites, current events, serial subtraction, counting and others. In addition to the TICS, we included four other cognitive tests: (1) immediate and (2) delayed recalls of the East Boston Memory Test (EBMT), (3) delayed recall of a 10-word list and (4) category fluency. Since we were particularly interested in verbal memory (as it is the strongest predictor of Alzheimer disease [12]), we included in our battery three specific tests of verbal memory. In the EBMT [6], a short paragraph is read and 12 key elements must be repeated immediately, and again after a 15-min delay. To further assess delayed verbal memory, we also added a delayed recall of the 10word list from the TICS (this addition is not part of the standard TICS and was not included in the TICS score). Finally, to test category fluency [13], women named as many animals as they could during $1 \mathrm{~min}$.

Increasingly, research on cognitive function utilizes composite scores, combining performance on several cognitive tests. Such composite scores integrate information from a variety of sources and thus provide a more stable representation of cognitive function than a single test. We calculated a verbal memory composite score by combining results of the immediate and delayed recalls of the EBMT [6] and the immediate and delayed recalls of the 10-word list. In addition, we calculated a summary composite score by combining all five cognitive tests: the TICS, the immediate and delayed recalls of the EBMT, the delayed word list recall, and verbal fluency. For both composite scores, relevant tests were combined by calculating z scores - taking the difference between the participant's score on each test and the mean, and dividing by the standard deviation.

The telephone cognitive assessments were administered by nurses trained to conduct these interviews. A study of inter-interviewer reliability demonstrated over $95 \%$ correlation for scoring across interviewers on each of the tests. In a validation study we conducted among similar highly-educated, high-functioning women from the Rush Religious Orders Study [12], we found a correlation of 0.81 comparing overall performance on our telephone-administered interview and performance on an in-person interview consisting of 21 tests, demonstrating high validity of our telephone method.

\section{Assessment of Education and Income Variables}

Participants reported information on educational attainment at enrollment into the WHS (1992-1996) when asked 'What is the highest level of education you have completed?' Response categories included: Licensed Practical Nurse/Licensed Vocational Nurse (LPN/LVN); 2-year Associate's degree/Registered Nurse (RN); nurse diploma program (3-year-RN); Bachelor's degree in nursing; Master's degree; and Doctoral degree (including a small percentage of physicians). For analysis, 3-year-RN was the reference group.

We also obtained information on participants' annual household income at enrollment. We asked 'Which of these income groups represents your total household income in the past year?' 
Women were asked to choose from under USD 10,000, USD 10,000-19,999, USD 20,000-29,999, USD 30,000-39,999, USD 40,000-49,999, USD 50,000-99,999, and USD 100,000 or more. For analysis, we combined the top two income categories, with the category of $\leq$ USD 19,999 as the reference group; 407 women who did not answer were excluded from the income analyses (in analyses of education, we included a missing variable for income when adjusting for income).

\section{Statistical Analysis}

In analyses of initial cognitive function, we used linear regression to obtain multivariable-adjusted mean differences in scores among those with different levels of education and income as well as their 95\% CIs. In addition, we used logistic regression models to estimate age- and multivariable-adjusted odds ratios (ORs) of cognitive impairment and corresponding 95\% CIs. For all tests, we defined cognitive impairment as the worst 10th percentile of our distribution, and compared these low-scorers with the remaining women. Such a population-based $10 \%$ cut-off point has high sensitivity and specificity for determining cognitive impairment [14].

To control for confounding in regression models, we included the following variables (in addition to the two primary variables of interest): age at interview (in years), history of diabetes (yes/no), history of high blood pressure (yes/no), history of depression (yes/ no), postmenopausal hormone use (never, current, past), body mass index $\left(<25,25-29.9,30+\mathrm{kg} / \mathrm{m}^{2}\right)$, smoking (current, past, never), physical activity (rare/never, $<1$ time/week, 1-3 times/week, 4+ times/week), and alcohol consumption ( $<1$ drink/month, 1-3 drink/month, 1-6 drink/week, 1+ drink/day). We did not adjust for cardiovascular disease as we had excluded cases of stroke, and there were few incident cases of heart disease at initial cognitive testing (prevalent cases of heart disease were not enrolled into this primary prevention trial). This information was provided at enrollment, when information on education and income was also collected (follow-up questionnaires between enrollment and initial cognitive testing did not include this same level of detail as the enrollment questionnaire).

In analyses of cognitive decline, we used linear regression to estimate multivariable-adjusted mean differences in decline. In addition, using logistic regression we obtained the ORs for substantial decline and their $95 \%$ CIs. We defined as cases of substantial decline those in the worst $10 \%$ of the distribution of change and as non-cases the remaining women. Covariates in models of cognitive decline included baseline test score and time between first and second interview as well as the variables described above.

\section{Results}

The majority of women in our sample had a 3-year RN diploma (46\%). Those with LPN/LVN or a 2-year RN represented 14 and $7 \%$, respectively; $18 \%$ of women had a bachelor's degree, $11 \%$ a master's degree, and $4 \%$ had a doctoral degree (table 1).

The average age of the women in each educational group was generally similar (table 1). As expected, the more educated women had higher household income, and were less likely to smoke cigarettes but more likely to report moderate alcohol intake. The women with higher educational attainment were also more likely to report lower body mass index and more frequent regular exercise, and had a somewhat lower prevalence of co-morbid conditions such as high blood pressure and type 2 diabetes.

\section{Analyses of Baseline Cognitive Function}

After adjustment for all these potential confounding factors, including income level, we found highly statistically significant trends of increasing mean cognitive performance with increasing education on the cognitive measures (table 2; p trends $<0.0005$ ). For example, on the TICS, mean score was higher by 0.8 points for those with a doctoral degree $(95 \%$ CI $0.5,1.2)$ compared with those having an RN diploma, whereas LPN/LVNs had a lower mean score (mean difference $=-0.5,95 \% \mathrm{CI}-0.7$, $-0.3)$.

For income, results were similar, although slightly weaker, with significant trends of increasing mean cognitive performance with increasing income on three of the four cognitive measures ( $p$ trends $<0.0001$ on TICS, verbal memory, summary score; $p$ trend $=0.8$ on verbal fluency). For example, on the TICS, mean score was higher by 0.7 points $(95 \%$ CI $0.5,1.0)$ for those with the highest income level ( $\geq$ USD 50,000) compared with the lowest income ( $\leq$ USD 19,999).

Findings were generally consistent when we examined odds of cognitive impairment (table 3). For example, there was a $30 \%$ lower risk of cognitive impairment on the TICS among women with a bachelor's degree compared with an $\mathrm{RN}$ diploma (OR $=0.7,95 \% \mathrm{CI} 0.6,1.0)$, while women with master's degree had a $40 \%$ decrease $(\mathrm{OR}=0.6,95 \% \mathrm{CI} 0.4,0.9)$, and women with a doctoral degree had a $50 \%$ decrease $(\mathrm{OR}=0.5,95 \%$ CI $0.3,0.9)$. In contrast, women with less education (LPN/LVN) had an $80 \%$ increased risk of cognitive impairment $(\mathrm{OR}=1.8$, $95 \%$ CI 1.4, 2.3), compared to those with a 3-year RN diploma. For income, there were 50\% lower risks of cognitive impairment on the TICS among women with a household income $\geq \mathrm{USD} 50,000$ (OR $=0.5,95 \% \mathrm{CI}, 0.3$, $0.9)$ compared with household income $<$ USD 19,999.

\section{Analyses of Cognitive Decline}

In analyses of cognitive decline, findings were generally consistent with those of initial cognitive function; however, results were somewhat weaker, likely due to the short period over which we measured decline (approximately 2 years). After multivariate adjustment, we found 
Table 1. Characteristics of WHS participants according to educational attainment

\begin{tabular}{|c|c|c|c|c|c|c|}
\hline & LPN/LVN & $\begin{array}{l}\text { 2-year } \\
\mathrm{AD} / \mathrm{RN}\end{array}$ & $\mathrm{RN}$ & $\mathrm{BA}$ or $\mathrm{BSc}$ & Masters & Doctorate \\
\hline $\mathrm{n}$ & 906 & 427 & 2,871 & 1,134 & 702 & 274 \\
\hline \multicolumn{6}{|l|}{ Mean age at initial cognitive assessment, } & 73.4 \\
\hline \multicolumn{7}{|l|}{ Household income, $\%{ }^{\mathrm{a}}$} \\
\hline$<$ USD 19,999 & 35.5 & 13.4 & 15.1 & 9.5 & 6.8 & 2.6 \\
\hline USD 20-29,999 & 29.4 & 19.9 & 23.9 & 15.6 & 13.1 & 5.8 \\
\hline USD 30-39,999 & 16.1 & 24.4 & 21.3 & 21.2 & 18.1 & 12.0 \\
\hline USD 40-49,999 & 8.2 & 14.5 & 14.1 & 18.3 & 18.1 & 11.3 \\
\hline USD 50,000 & 3.1 & 23.2 & 18.5 & 29.3 & 37.8 & 62.4 \\
\hline \multicolumn{7}{|l|}{ Cigarette smoking, $\%{ }^{\mathrm{b}}$} \\
\hline Current & 13.4 & 11.2 & 10.7 & 7.5 & 6.3 & 6.6 \\
\hline Past & 33.4 & 33.0 & 40.0 & 36.7 & 37.5 & 38.3 \\
\hline Never & 53.0 & 55.5 & 49.4 & 55.6 & 56.3 & 55.1 \\
\hline Alcohol consumption $\geq 1 \mathrm{drink} / \mathrm{day}, \%$ & 4.4 & 10.3 & 13.0 & 13.4 & 14.8 & 21.2 \\
\hline $\mathrm{BMI} \geq 30 \mathrm{~kg} / \mathrm{m}^{2}, \%$ & 20.3 & 16.6 & 14.4 & 12.6 & 13.7 & 8.8 \\
\hline Exercise $\geq 4$ times/week, $\%$ & 8.5 & 9.8 & 12.1 & 12.4 & 15.0 & 15.3 \\
\hline \multicolumn{7}{|l|}{ Current use of postmenopausal } \\
\hline hormones, $\%$ & 30.9 & 42.2 & 37.9 & 42.1 & 46.2 & 38.3 \\
\hline High blood pressure, $\%$ & 44.6 & 40.3 & 40.1 & 38.0 & 38.5 & 32.5 \\
\hline Diabetes mellitus, $\%$ & 4.5 & 5.2 & 3.2 & 3.2 & 2.7 & 2.6 \\
\hline History of depression, $\%$ & 6.1 & 6.6 & 5.5 & 5.5 & 6.4 & 6.9 \\
\hline
\end{tabular}

${ }^{a}$ These income categories do not add up to $100 \%$ because of missing data on income from 407 women.

${ }^{\mathrm{b}}$ Current, past, and never smokers do not add up to $100 \%$ because of missing data on smoking from 11 women.

Table 2. Mean differences in cognitive function by educational attainment and household income: WHS

\begin{tabular}{|c|c|c|c|c|c|c|c|c|}
\hline & \multicolumn{8}{|l|}{ Mean difference } \\
\hline & \multicolumn{2}{|l|}{ TICS } & \multicolumn{2}{|l|}{ verbal fluency } & \multicolumn{2}{|l|}{ verbal memory } & \multicolumn{2}{|l|}{ summary score } \\
\hline & age adjusted & $\begin{array}{l}\text { multi-variable } \\
\text { adjusted }\end{array}$ & age adjusted & $\begin{array}{l}\text { multi-variable } \\
\text { adjusted }\end{array}$ & age adjusted & $\begin{array}{l}\text { multi-variable } \\
\text { adjusted }\end{array}$ & age adjusted & $\begin{array}{l}\text { multi-variable } \\
\text { adjusted }\end{array}$ \\
\hline \multicolumn{9}{|l|}{ Education } \\
\hline LPN/LVN & $0.7(-0.9,-0.5)$ & $-0.5(-0.7,-0.3)$ & $-0.8(-1.2,-0.4)$ & $-0.6(-1.0,-0.2)$ & $-0.1(-0.2,-0.1)$ & $-0.1(-0.1,-0.04)$ & $-0.2(-0.2,-0.1)$ & $-0.1(-0.2,-0.1)$ \\
\hline 2-year AD/RN & $-0.03(-0.2,0.3)$ & $0.1(-0.2,0.3)$ & $-0.1(-0.6,0.4)$ & $-0.1(-0.6,0.4)$ & $-0.01(-0.1,0.04)$ & $-0.01(-0.1,0.1)$ & $-0.02(-0.1,0.04)$ & $-0.01(-0.1,0.1)$ \\
\hline $\mathrm{RN}$ & 0.0 (ref) & 0.0 (ref) & $0.0(\mathrm{ref})$ & $0.0(\mathrm{ref})$ & 0.0 (ref) & 0.0 (ref) & 0.0 (ref) & 0.0 (ref) \\
\hline $\mathrm{BA}$ or BSc & $0.5(0.3,0.6)$ & $0.4(0.2,0.6)$ & $0.7(0.3,1.0)$ & $0.6(0.3,0.9)$ & $0.04(-0.002,0.1)$ & $0.03(-0.01,0.1)$ & $0.1(-0.04,0.1)$ & $0.1(0.03,0.1)$ \\
\hline Masters & $0.6(0.4,0.8)$ & $0.5(0.3,0.7)$ & $0.4(0.03,0.8)$ & $0.3(-0.1,0.7)$ & $0.1(0.02,0.1)$ & $0.1(0.004,0.1)$ & $0.1(0.06,0.2)$ & $0.1(0.04,0.1)$ \\
\hline Doctorate & $1.1(0.7,1.4)$ & $0.8(0.5,1.2)$ & $1.7(1.1,2.3)$ & $1.6(1.0,2.2)$ & $0.2(0.1,0.2)$ & $0.1(0.04,0.2)$ & $0.2(0.2,0.3)$ & $0.2(0.1,0.3)$ \\
\hline $\mathrm{p}$ for trend & & 0.0005 & & 0.0001 & & 0.0004 & & 0.0005 \\
\hline \multicolumn{9}{|l|}{ Income, USD } \\
\hline 19,999 & $0.0(\mathrm{ref})$ & 0.0 (ref) & 0.0 (ref) & 0.0 (ref) & 0.0 (ref) & 0.0 (ref) & 0.0 (ref) & 0.0 (ref) \\
\hline $20-29,999$ & $0.5(0.3,0.8)$ & $0.4(0.2,0.6)$ & $0.6(0.2,1.0)$ & $0.4(-0.05,0.8)$ & $0.1(0.04,0.1)$ & $0.1(0.02,0.1)$ & $0.1(0.1,0.2)$ & $0.1(0.04,0.1)$ \\
\hline $30-39,999$ & $0.7(0.5,0.9)$ & $0.4(0.2,0.7)$ & $1.1(0.7,1.5)$ & $0.7(0.2,1.1)$ & $0.1(0.1,0.2)$ & $0.1(0.04,0.1)$ & $0.2(0.1,0.2)$ & $0.1(0.1,0.2)$ \\
\hline $40-49,999$ & $0.9(0.7,1.2)$ & $0.6(0.3,0.8)$ & $1.1(0.6,1.5)$ & $0.6(0.1,1.1)$ & $0.2(0.1,0.2)$ & $0.1(0.1,0.2)$ & $0.2(0.2,0.3)$ & $0.1(0.1,0.2)$ \\
\hline$\geq 50,000$ & $1.2(1.0,1.4)$ & $0.7(0.5,1.0)$ & $1.0(0.6,1.4)$ & $0.3(-0.2,0.7)$ & $0.2(0.1,0.2)$ & $0.1(0.1,0.2)$ & $0.2(0.2,0.3)$ & $0.1(0.1,0.2)$ \\
\hline $\mathrm{p}$ for trend & & $<0.0001$ & & 0.8 & & $<0.0001$ & & $<0.0001$ \\
\hline
\end{tabular}

Models include age at interview, cigarette smoking, alcohol intake, BMI, physical activity, high blood pressure, diabetes, postmenopausal hormone use, history of depression, and education and income. 
Table 3. Odds of impaired cognitive function according to educational attainment and household income: WHS

\begin{tabular}{|c|c|c|c|c|c|c|c|c|c|c|c|c|}
\hline & \multicolumn{12}{|c|}{ Odds ratio } \\
\hline & \multicolumn{3}{|c|}{ TICS } & \multicolumn{3}{|c|}{ verbal fluency } & \multicolumn{3}{|c|}{ verbal memory } & \multicolumn{3}{|c|}{ summary score } \\
\hline & cases & age adjusted & $\begin{array}{l}\text { multi-variable } \\
\text { adjusted }\end{array}$ & cases & age adjusted & $\begin{array}{l}\text { multi-variable } \\
\text { adjusted }\end{array}$ & cases & age adjusted & $\begin{array}{l}\text { multi-variable } \\
\text { adjusted }\end{array}$ & cases & age adjusted & $\begin{array}{l}\text { multi-variable } \\
\text { adjusted }\end{array}$ \\
\hline \multicolumn{13}{|l|}{ Education } \\
\hline LPN/LVN & 137 & $2.0(1.6-2.5)$ & $1.8(1.4-2.3)$ & 123 & $1.5(1.2-1.9)$ & $1.4(1.1-1.8)$ & 139 & $1.8(1.5-2.3)$ & $1.6(1.3-2.0)$ & 143 & $1.9(1.5-2.4)$ & $1.6(1.3-2.0)$ \\
\hline 2-year AD/RN & 31 & $1.0(0.7-1.5)$ & $1.0(0.7-1.5)$ & 47 & $1.3(0.9-1.8)$ & $1.3(0.9-1.8)$ & 41 & $1.2(0.8-1.7)$ & $1.2(0.9-1.7)$ & 44 & $1.3(0.9-1.8)$ & $1.3(0.9-1.9)$ \\
\hline $\mathrm{RN}$ & 243 & 1.0 (ref) & 1.0 (ref) & 280 & 1.0 (ref) & 1.0 (ref) & 273 & 1.0 (ref) & 1.0 (ref) & 280 & 1.0 (ref) & 1.0 (ref) \\
\hline $\mathrm{BA}$ or $\mathrm{BSc}$ & 65 & $0.7(0.5-0.9)$ & $0.7(0.6-1.0)$ & 112 & $1.1(0.8-1.3)$ & $1.1(0.9-1.4)$ & 104 & $1.0(0.8-1.3)$ & $1.1(0.8-1.4)$ & 91 & $0.9(0.7-1.1)$ & $0.9(0.7-1.2)$ \\
\hline Masters & 35 & $0.6(0.4-0.8)$ & $0.6(0.4-0.9)$ & 68 & $1.0(0.8-1.4)$ & $1.0(0.8-1.4)$ & 54 & $0.8(0.6-1.1)$ & $0.9(0.6-1.2)$ & 53 & $0.8(0.6-1.0)$ & $0.8(0.6-1.1)$ \\
\hline Doctorate & 12 & $0.5(0.3-0.9)$ & $0.5(0.3-0.9)$ & 23 & $0.7(0.5-1.1)$ & $0.8(0.5-1.2)$ & 21 & $0.6(0.4-1.0)$ & $0.7(0.5-1.2)$ & 18 & $0.5(0.3-0.8)$ & $0.6(0.3-0.9)$ \\
\hline $\mathrm{p}$ for trend & & & $<0.0001$ & & & 0.02 & & & $<0.0001$ & & & $<0.0001$ \\
\hline \multicolumn{13}{|l|}{ Income, USD } \\
\hline$\leq 19,999$ & 146 & 1.0 (ref) & 1.0 (ref) & 139 & 1.0 (ref) & 1.0 (ref) & 157 & 1.0 (ref) & 1.0 (ref) & 176 & 1.0 (ref) & 1.0 (ref) \\
\hline $20-29,999$ & 126 & $0.7(0.5-0.8)$ & $0.7(0.6-1.0)$ & 131 & $0.7(0.6-0.9)$ & $0.8(0.6-1.0)$ & 164 & $0.8(0.6-1.0)$ & $0.9(0.7-1.1)$ & 149 & $0.6(0.5-0.8)$ & $0.7(0.5-0.9)$ \\
\hline $30-39,999$ & 97 & $0.6(0.4-0.7)$ & $0.7(0.5-1.0)$ & 121 & $0.7(0.6-1.0)$ & $0.9(0.6-1.1)$ & 118 & $0.7(0.5-0.9)$ & $0.8(0.6-1.0)$ & 116 & $0.6(0.4-0.7)$ & $0.7(0.5-0.9)$ \\
\hline $40-49,999$ & 61 & $0.5(0.4-0.7)$ & $0.7(0.5-1.0)$ & 94 & $0.9(0.6-1.1)$ & $1.0(0.7-1.4)$ & 72 & $0.6(0.4-0.8)$ & $0.7(0.5-1.0)$ & 66 & $0.5(0.4-0.7)$ & $0.6(0.4-0.8)$ \\
\hline$\geq 50,000$ & 61 & $0.3(0.2-0.4)$ & $0.5(0.4-0.7)$ & 122 & $0.7(0.5-0.9)$ & $0.9(0.7-1.2)$ & 93 & $0.5(0.4-0.7)$ & $0.6(0.5-0.8)$ & 88 & $0.4(0.3-0.6)$ & $0.6(0.4-0.8)$ \\
\hline $\mathrm{p}$ for trend & & & $<0.0001$ & & & 0.97 & & & 0.003 & & & 0.003 \\
\hline
\end{tabular}

Models include age at interview, smoking, alcohol intake, BMI, physical activity, high blood pressure, diabetes, postmenopausal hormone use, history of depression, and education and income.

statistically significant trends of lower rates of mean decline with increasing educational attainment on three of four cognitive measures (table 4; p trends $<0.003$ on TICS, verbal fluency, and summary score, $\mathrm{p}$ trend $=0.5$ on verbal memory). For income, significant trends of lower rates of decline with increasing income were observed on two of the four cognitive measures ( $p$ trend $<0.0001$ on TICS; $p$ trend $=0.5$ on verbal fluency; $p$ trend $=0.02$ on verbal memory; $\mathrm{p}$ trend $=0.1$ on summary score).

When we examined the odds of substantial cognitive decline over time (table 5), we also found lower risk of decline with higher levels of education, with statistically significant results on two of the four cognitive measures. For example, comparing women with a doctoral degree to those with an RN diploma, the ORs were $0.4(95 \% \mathrm{CI}$ $0.2,0.7)$ on the TICS, $0.5(95 \%$ CI $0.3,0.8)$ on verbal fluency, $0.8(95 \%$ CI $0.5,1.2)$ on verbal memory, and 0.7 $(95 \%$ CI $0.4-1.2)$ on the summary score. For income, ORs were generally below 1.0 for higher levels of income, however, few results achieved statistical significance.

Finally, we examined interactions between education and income for both baseline cognitive function and cognitive decline. However, we found no significant interactions.

Education, Income, and Cognitive Function

\section{Discussion}

After adjusting for numerous health and lifestyle factors, we found trends of better cognitive function and less cognitive decline with both increasing education and increasing annual income level among community-dwelling health professionals aged 66 years or older. All these women had at least 1-2 years of education after high school.

There are several important strengths of this study. First, most previous investigations have focused on populations with substantially lower levels of education, while we were able to provide information on the effects of education and income at the higher end of the spectrum. Although we previously examined education and income in the Nurses' Health Study, another population of health professionals, the range of educational attainment was somewhat narrower in that group, as the lowest educational level was a 3-year RN diploma, whereas this Women's Health Study cohort also included women with 1 year of education after high school. In addition, income information in the current study is from individual household income data, whereas that of the Nurses' Health Study was from the census tract median household income data and was thus likely less accurate. Finally, in

Neuroepidemiology 2006;26:93-101 
Table 4. Mean differences in cognitive decline scores by educational attainment and household income: WHS

\begin{tabular}{|c|c|c|c|c|c|c|c|c|}
\hline & \multicolumn{8}{|l|}{ Mean difference } \\
\hline & \multicolumn{2}{|l|}{ TICS } & \multicolumn{2}{|l|}{ verbal fluency } & \multicolumn{2}{|l|}{ verbal memory } & \multicolumn{2}{|l|}{ summary score } \\
\hline & age adjusted & $\begin{array}{l}\text { multi-variable } \\
\text { adjusted }\end{array}$ & age adjusted & $\begin{array}{l}\text { multi-variable } \\
\text { adjusted }\end{array}$ & age adjusted & $\begin{array}{l}\text { multi-variable } \\
\text { adjusted }\end{array}$ & age adjusted & $\begin{array}{l}\text { multi-variable } \\
\text { adjusted }\end{array}$ \\
\hline \multicolumn{9}{|l|}{ Education } \\
\hline $\begin{array}{l}\text { LPN/LVN } \\
\text { 2-year }\end{array}$ & $-0.6(-0.8,-0.4)$ & $-0.5(-0.7,-0.2)$ & $-0.7(-1.0,-0.3)$ & $-0.6(-0.9,-0.2)$ & $-0.04(-0.1,0.02)$ & $-0.01(-0.1,0.1)$ & $-0.1(-0.1,-0.01)$ & $-0.04(-0.1,0.01)$ \\
\hline $\mathrm{AD} / \mathrm{RN}$ & $-0.1(-0.4,0.2)$ & $-0.1(-0.4,0.2)$ & $-0.1(-0.5,0.4)$ & $-0.01(-0.5,0.5)$ & $0.02(-0.1,0.1)$ & $0.02(-0.1,0.1)$ & $0.01(-0.1,0.1)$ & $0.02(-0.1,0.1)$ \\
\hline $\mathrm{RN}$ & 0.0 (ref) & 0.0 (ref) & 0.0 (ref) & 0.0 (ref) & 0.0 (ref) & 0.0 (ref) & 0.0 (ref) & 0.0 (ref) \\
\hline $\mathrm{BA}$ or $\mathrm{BSc}$ & $0.1(-0.1,0.3)$ & $0.03(-0.2,0.2)$ & $0.6(0.2,0.9)$ & $0.5(0.2,0.9)$ & $-0.0003(-0.1,0.1)$ & $-0.01(-0.1,0.1)$ & $0.02(-0.03,0.1)$ & $0.01(-0.03,0.1)$ \\
\hline Masters & $0.1(-0.1,0.4)$ & $0.1(-0.2,0.3)$ & $0.4(-0.04,0.7)$ & $0.3(-0.1,0.7)$ & $-0.01(-0.1,0.1)$ & $-0.02(-0.1,0.04)$ & $-0.002(-0.05,0.1)$ & $-0.01(-0.1,0.04)$ \\
\hline Doctorate & $0.9(0.6,1.3)$ & $0.8(0.4,1.1)$ & $1.7(1.1,2.3)$ & $1.6(1.0,2.2)$ & $0.1(-0.004,0.2)$ & $0.1(-0.02,0.2)$ & $0.2(0.1,0.2)$ & $0.1(0.1,0.2)$ \\
\hline $\mathrm{p}$ for trend & & $<0.0001$ & & $<0.0001$ & & 0.5 & & 0.003 \\
\hline \multicolumn{9}{|l|}{ Income, USD } \\
\hline$\leq 19,999$ & 0.0 (ref) & 0.0 (ref) & 0.0 (ref) & 0.0 (ref) & 0.0 (ref) & 0.0 (ref) & 0.0 (ref) & 0.0 (ref) \\
\hline 20-29,999 & $0.4(0.2,0.6)$ & $0.3(0.1,0.6)$ & $0.2(-0.2,0.6)$ & $0.1(-0.3,0.5)$ & $0.1(0.01,0.1)$ & $0.1(0.01,0.1)$ & $0.1(0.01,0.1)$ & $0.1(0.003,0.1)$ \\
\hline $30-39,999$ & $0.7(0.5,0.9)$ & $0.5(0.3,0.8)$ & $0.4(-0.02,0.8)$ & $0.1(-0.3,0.5)$ & $0.1(0.04,0.2)$ & $0.1(0.04,0.2)$ & $0.1(0.04,0.2)$ & $0.1(0.03,0.1)$ \\
\hline $40-49,999$ & $0.5(0.2,0.7)$ & $0.2(-0.02,0.5)$ & $0.4(-0.1,0.8)$ & $0.01(-0.4,0.5)$ & $0.1(0.01,0.1)$ & $0.1(-0.004,0.1)$ & $0.1(0.01,0.1)$ & $0.04(-0.02,0.1)$ \\
\hline$\geq 50,000$ & $0.9(0.7,1.1)$ & $0.6(0.3,0.8)$ & $0.7(0.3,1.1)$ & $0.2(-0.3,0.6)$ & $0.1(0.1,0.2)$ & $0.1(0.04,0.2)$ & $0.1(0.1,0.2)$ & $0.1(0.02,0.1)$ \\
\hline $\mathrm{p}$ for trend & & $<0.0001$ & & 0.5 & & 0.02 & & 0.1 \\
\hline
\end{tabular}

Models include age at interview, cigarette smoking, alcohol intake, BMI, physical activity, high blood pressure, diabetes, postmenopausal hormone use, history of depression, and education and income.

Table 5. Odds of substantial cognitive decline according to educational attainment and household income: WHS

\begin{tabular}{|c|c|c|c|c|c|c|c|c|c|c|c|c|}
\hline & \multicolumn{12}{|c|}{ Odds ratio } \\
\hline & \multicolumn{3}{|l|}{ TICS } & \multicolumn{3}{|c|}{ verbal fluency } & \multicolumn{3}{|c|}{ verbal memory } & \multicolumn{3}{|c|}{ summary score } \\
\hline & cases & age adjusted & $\begin{array}{l}\text { multi-variable } \\
\text { adjusted }\end{array}$ & cases & age adjusted & $\begin{array}{l}\text { multi-variable } \\
\text { adjusted }\end{array}$ & cases & age adjusted & $\begin{array}{l}\text { multi-variable } \\
\text { adjusted }\end{array}$ & cases & age adjusted & $\begin{array}{l}\text { multi-variable } \\
\text { adjusted }\end{array}$ \\
\hline \multicolumn{13}{|l|}{ Education } \\
\hline LPN/LVN & 93 & $1.5(1.2-2.0)$ & $1.4(1.1-1.9)$ & 75 & $1.1(0.8-1.5)$ & $1.0(0.8-1.5)$ & 69 & $1.0(0.8-1.4)$ & $1.0(0.7-1.3)$ & 76 & $1.2(0.9-1.5)$ & $1.1(0.8-1.5)$ \\
\hline 2-year AD/RN & 39 & $1.2(0.8-1.7)$ & $1.2(0.8-1.8)$ & 49 & $1.3(0.9-1.8)$ & $1.2(0.9-1.8)$ & 27 & $0.8(0.5-1.1)$ & $0.8(0.5-1.2)$ & 33 & $0.9(0.6-1.4)$ & $0.9(0.6-1.4)$ \\
\hline $\mathrm{RN}$ & 248 & $1.0(\mathrm{ref})$ & $1.0(\mathrm{ref})$ & 276 & $1.0(\mathrm{ref})$ & $1.0(\mathrm{ref})$ & 247 & $1.0(\mathrm{ref})$ & $1.0(\mathrm{ref})$ & 250 & $1.0(\mathrm{ref})$ & 1.0 (ref) \\
\hline $\mathrm{BA}$ or $\mathrm{BSc}$ & 103 & $0.9(0.7-1.2)$ & $1.0(0.7-1.2)$ & 115 & $0.9(0.7-1.1)$ & $0.9(0.7-1.1)$ & 115 & $1.1(0.9-1.4)$ & $1.1(0.9-1.4)$ & 107 & $1.0(0.8-1.3)$ & $1.0(0.8-1.3)$ \\
\hline Masters & 69 & $1.0(0.7-1.3)$ & $1.0(0.7-1.3)$ & 67 & $0.8(0.6-1.1)$ & $0.9(0.6-1.2)$ & 72 & $1.1(0.8-1.4)$ & $1.1(0.8-1.5)$ & 68 & $1.0(0.8-1.4)$ & $1.0(0.8-1.4)$ \\
\hline Doctorate & 17 & $0.4(0.3-0.7)$ & $0.4(0.2-0.7)$ & 17 & $0.4(0.2-0.8)$ & $0.5(0.3-0.8)$ & 26 & $0.8(0.5-1.3)$ & $0.8(0.5-1.2)$ & 23 & $0.7(0.5-1.1)$ & $0.7(0.4-1.2)$ \\
\hline $\mathrm{p}$ for trend & & & 0.0002 & & & 0.002 & & & 0.9 & & & 0.3 \\
\hline \multicolumn{13}{|l|}{ Income, USD } \\
\hline$\leq 19,999$ & 94 & 1.0 (ref) & 1.0 (ref) & 81 & 1.0 (ref) & 1.0 (ref) & 95 & 1.0 (ref) & 1.0 (ref) & 99 & 1.0 (ref) & 1.0 (ref) \\
\hline $20-29,999$ & 118 & $0.8(0.6-1.1)$ & $0.9(0.7-1.2)$ & 112 & $0.9(0.6-1.2)$ & $0.9(0.7-1.3)$ & 99 & $0.7(0.5-0.9)$ & $0.6(0.5-0.9)$ & 113 & $0.8(0.6-1.0)$ & $0.7(0.6-1.0)$ \\
\hline $30-39,999$ & 106 & $0.8(0.6-1.1)$ & $0.9(0.6-1.2)$ & 134 & $1.0(0.7-1.3)$ & $1.0(0.7-1.4)$ & 102 & $0.7(0.5-1.0)$ & $0.7(0.5-1.0)$ & 93 & $0.6(0.5-0.9)$ & $0.6(0.5-0.9)$ \\
\hline 40-49,999 & 93 & $0.9(0.7-1.3)$ & $1.1(0.8-1.6)$ & 100 & $1.0(0.7-1.4)$ & $1.1(0.8-1.6)$ & 96 & $1.0(0.7-1.3)$ & $0.9(0.7-1.3)$ & 95 & $1.0(0.7-1.3)$ & $1.0(0.7-1.4)$ \\
\hline$\geq 50,000$ & 114 & $0.6(0.5-0.9)$ & $0.8(0.6-1.2)$ & 126 & $0.7(0.5-1.0)$ & $0.9(0.6-1.3)$ & 128 & $0.7(0.6-1.0)$ & $0.7(0.5-1.0)$ & 118 & $0.7(0.5-1.0)$ & $0.8(0.6-1.1)$ \\
\hline $\mathrm{p}$ for trend & & & 0.5 & & & 0.5 & & & 0.4 & & & 0.5 \\
\hline
\end{tabular}

Models include age at interview, cigarette smoking, alcohol intake, BMI, physical activity, high blood pressure, diabetes, postmenopausal hormone use, history of depression, and education and income. 
this population of health professionals, several important confounding variables, such as healthcare access, are likely minimized; furthermore, the extensive information on a wide array of health and lifestyle variables allowed careful control for numerous potential confounding factors which may have important effects on the apparent relation between education/socioeconomic status and cognitive function.

There are some limitations to our study. It is possible that we underestimated effects of socioeconomic status if income alone insufficiently characterizes the full extent of a participant's economic situation; in particular, household income may imperfectly represent socioeconomic status if the women or their spouses were retired (participants were generally in their sixties at trial enrollment). In this observational study, we could not fully control for confounding, and there may be unmeasured confounding factors, or residual confounding, which could partly influence our observed associations; this should be considered in interpreting our results. Such uncontrolled confounding would likely result in overestimates of the true associations between education, income and cognition as women with higher education or income were generally healthier; nonetheless, since we observed relatively minor attenuation of our effect estimates after adjusting for numerous potential covariates, it is unlikely that uncontrolled confounding could completely explain the strong relations we observed between education or income and cognition. Lastly, we administered a brief telephone cognitive assessment, and thus cannot measure all aspects of cognition. We chose to focus our test battery on verbal memory because verbal memory is among the strongest predictors of eventual development of Alzheimer disease [13], and we thus address a major public health issue.

Similar to our findings, other large studies have generally reported beneficial effects of educational attainment on cognitive function among subjects with somewhat less education than ours. In cross-sectional studies of men, both Gallacher et al. [4] in the Caerphilly Study and Cagney and Lauderdale [5] found a significant trend of increasing cognitive scores with increasing education after adjustment for age and socioeconomic status. In a prospective study of 3,266 community-dwelling subjects aged over 65 years, Evans et al. [2] found that each level of education (ranging from 0 to $12+$ years) predicted significantly less cognitive decline after multivariate adjustment. Another study observed 70\% lower risk of decline on the MMSE with increments in education (education categorized as $<10$ years, $10-12$ years, or some college) in
14,833 subjects aged 18 years and older [1]. A study with longer follow-up (11.5 years) of 1,488 men and women aged 18 years and older also found that more than 8 years of formal education was associated with significantly less decline on the MMSE, after adjusting for age and race [3]. In a recent study of 557 community-dwelling Spanish men and women over aged 65 years, less education (no primary school education vs. primary school) was associated with increased odds of cognitive decline over 4 years $(\mathrm{OR}=2.36,95 \%$ CI 1.16, 4.81) [7]. More recently, Seeman et al. [9], after following 895 community-dwelling men and women for 3-7 years, observed increasingly better cognitive performance on tests of memory, naming, spatial recognition, figures/copying, and similarities with increasing educational attainment (categorized as $0-8$ years, 9-11 years, 12 years, and college or more) increased [9]. Finally, in the Nurses' Health Study of 19,319 community-dwelling women aged 70-79 years, Lee et al. [8] found a significantly decreased risk of cognitive impairment $(\mathrm{OR}=0.49,95 \% \mathrm{CI} 0.36,0.66)$ and of cognitive decline $(\mathrm{RR}=0.65,95 \% \mathrm{CI} 0.50,0.86)$ in women with a graduate degree compared with an RN diploma, after adjusting for potential confounders. Thus the data reported here serve as important confirmation of the cognitive impact of education, even among well-educated subjects.

There are several possible mechanisms which can explain the association of education to cognitive function. First, education may simply reflect better health or other behavioral choices that in turn lead to maintenance of cognitive function [15]. That is, the relation between education and cognition may be largely due to confounding. However, our finding that effects of education persisted after carefully adjusting for numerous health and behavioral characteristics in a population of health professionals, relatively homogeneous in their health habits and access to healthcare, suggests that confounding by health status is not the most likely explanation. Secondly, higher levels of education may improve an individual's ability to perform on tests of cognitive function or to hide mild cognitive deficits [15]. Again, in our group of educated professionals, it does not seem likely that this could completely explain the substantial differences in performance that we observed between women who are all highly educated.

Thus our data suggest it is most likely that education exerts direct effects on brain structure or function. One hypothesis proposes that education may act early in life by increasing synapse number or vascularization and creating cognitive reserves [15]. This has been called the 'reserve capacity' hypothesis, in which it is believed that 
those with a greater cognitive reserve can tolerate more brain disturbance before manifesting cognitive symptoms. Interestingly, a recent study indicated that level of education modulated the effects of cerebral white matter hyperintensities on cognition [16]; with significant associations between severe white matter hyperintensities and lower cognitive performances in participants with lower levels of education but not in those with higher education. Another study also suggested that the relation of neuritic and diffuse plaques to cognition was modified by level of education [17]. An additional hypothesis is that education in early life may have effects in later life, if those with more education continue searching for mental stimulation (the 'use it or lose it' hypothesis), possibly leading to beneficial neurochemical or structural alterations in the brain $[15,18]$. Indeed, several studies have found that mental stimulation from social or leisure activities $[19,20]$ was associated with decreased incidence of cognitive decline and dementia [21,22].

Fewer research has addressed the effect of income on cognitive function, and results have not been entirely consistent. In their cross-sectional study, Cagney and Lauderdale [5] reported that, compared with those whose yearly income was greater than USD 50,000, those with income below USD 5,000 had lower scores by $0.36-0.98$ points $(p<0.05)$ in tests of memory, working memory, knowledge, language, and orientation. Net worth, consisting of value of home owned, checking or savings accounts, etc., had greater effects than income. In a prospective study, Evans et al. [2] reported that each USD 5,000 increase in income predicted a 0.03 -point increase in im- mediate memory score $(p=0.04)$, but they found no effect of income on the results of a mental status test. In a largescale investigation of women with more than high school education, there was no effect of census tract median household income [8], although this is a relatively crude measure of income. Findings from our current study generally support a relation between household income and cognitive function. However, further study using alternate measures of socioeconomic status (e.g., net worth) may be useful in better evaluating this relationship since household income alone may not fully represent socioeconomic status, especially in older persons. Moreover, the greater attenuation of effect estimates we found after multivariable adjustment in analyses of income than of education suggests that apparent relations with income may be partly mediated by health and lifestyle factors; thus, further research regarding mechanisms explaining the relation between income and cognition will be important.

Overall, we found clear relations between education, income, and cognitive function. In particular, this may point to feasible means for reducing cognitive decline if the relation with education might be explained by increased cognitive reserve or increased mental stimulation in those with higher levels of education.

\section{Acknowledgment}

This study was supported by grants AG15933, HL 43851, and CA 47988 from the National Institutes of Health.

\section{References}

1 Farmer ME, Kittner SJ, Rae DS, Bartko JJ, Regier DA: Education and change in cognitive function. Ann Epidemiol 1995;5:1-7.

-2 Evans DA, Beckett LA, Albert MS, Hebert LE, Scherr PA, Funkenstein HH, Taylor JO: Level of education and change in cognitive function in a community population of older persons. Ann Epidemiol 1993;3:71-77.

-3 Lyketsos CG, Chen L-S, Anthony JC: Cognitive decline in adulthood: an 11.5-year followup of the Baltimore Epidemiologic Catchment Area Study. Am J Psychiatry 1999;156:5865.

4 Gallacher JE, Elwood PC, Hopkinson C, Patrick MA, Rabbitt PMA, Stollery BT, Sweetnam PM, Brayne C, Huppert FA: Cognitive function in the Caerphilly study: associations with age, social class, education and mood. Eur J Epidemiol 1999;15:161-169.
5 Cagney KA, Lauderdale DS: Education, wealth, and cognitive function in later life. $\mathrm{J}$ Gerontol Psychol Sci 2002;57B:163-172.

-6 Scherr P, Albert M, Funkenstein H, Cook N, Hennekens C, Branch L, White L, Taylor J, Evans D: Correlates of cognitive function in an elderly community population. Am J Epidemiol 1988;128:1084-1101.

7 Alvarado BE, Zunzunegui M-V, Del Ser T, and Beland F: Cognitive decline is related to education and occupation in a Spanish elderly cohort. Aging Clin Exp Res 2002;14:132-142.

-8 Lee S, Kawachi I, Berkman LF, Grodstein F: Education, other socioeconomic indicators, and cognitive function in older women. Am $\mathrm{J}$ Epidemiol 2003;157:712-720.
9 Seeman TE, Huang M, Bretsky P, Crimmins E, Launer L, Guralnik JM: Education and APOE-e4 in longitudinal cognitive decline: MacArthur Studies of Successful Aging. J Gerontol B Psychol Sci Soc Sci 2005;60:P74P83.

10 Buring JE, Hennekens CH: The Women's Health Study: Summary of the Study Design. J Myocardial Ischemia 1992;4:27-29.

11 Brandt J, Spencer M, Folstein MF: The telephone interview for cognitive status. Neuropsychiatry Neuropsychol Behav Neurol 1988; 1:111-117.

12 Mufson EJ, Chen E-Y, Cochran EJ, Beckett LA, Bennett DA, Kordower JH: Entorhinal cortex beta-amyloid load in individuals with mild cognitive impairment. Exp Neurol 1999; 158:469-490. 
13 Morris JC, Heyman A, Mohs RC, Hughes JP, van Belle $G$, Fillenbaum G, Mellits ED, Clark $\mathrm{C}$ : The consortium to establish a registry for Alzheimer's disease (CERAD). Part 1. Clinical and neuropsychological assessment of Alzheimer's disease. Neurology 1989;39:11591165.

14 Ganguli M: The use of screening instruments for the detection of dementia. Neuroepidemiology 1997; 16:271-280.

15 Albert MS: How does education affect cognitive function? Ann Epidemiol 1995;5:76-78.

$\checkmark 16$ Dufouil C, Alperovitch A, Tzourio C: Influence of education on the relationship between white matter lesions and cognition. Neurology 2003; 60:831-836.
7 Bennett DA, Wilson RS, Schneider JA, Evans DA, Mendes de Leon CF, Arnold SE, Barnes LL, Bienias JL: Education modifies the relation of AD pathology to level of cognitive function in older persons. Neurology 2003;60: 1909-1915.

18 Scarmeas N, Zarahn E, Andersen KE, Habeck CG, Hilton J, Flynn J, Marmot MG, Bell KL, Sackeim HA, Van Heertum RL, Moeller JR, Stern Y: Association of life activities with cerebral blood flow in Alzheimer disease. Arch Neurol 2003;60:359-365.

19 Wilson RS, Bennett DA, Beckett LA, Morris MC, Gilley DW, Bienias JL, Scherr PA, Evans DA: Cognitive activity in older persons from a geographically defined population. J Gerontol B Psychol Sci Soc Sci 1999;54:P155-P160.
20 Kliegel M, Zimprich D, Rott C: Life-long intellectual activities mediate the predictive effect of early education on cognitive impairment in centenarians: a retrospective study. Aging Men Health 2004;8:430-437.

21 Wang H, Karp A, Winbald B, Fratiglioni L: Late-life engagement in social and leisure activities is associated with a decreased risk of dementia: a longitudinal study from the Kungsholmen Project. Am J Epidemiol 2002; 155:1081-1087.

-22 Verghese J, Lipton RB, Katz MJ, Hall CB, Derby CA, Kuslansky G, Ambrose AF, Sliwinski $\mathrm{M}$, Buschke H: Leisure activities and the risk of dementia in the elderly. N Engl J Med 2003; 348:2508-2516. 